\title{
FACILE FABRICATION OF LOW-COST PASSIVE WIRELESS HUMIDITY SENSOR FOR SMART PACKAGING VIA ALL-LASER PROCESSING OF METALIZED PAPER
}

\author{
Rahim Rahimi ${ }^{1,2}$, Jiawei Zhou ${ }^{1,2}$, Hongjie Jiang ${ }^{1,2}$, Tahereh Soleimani ${ }^{3}$, and Babak Ziaie ${ }^{1,2 *}$ \\ ${ }^{1}$ Purdue University, West Lafayette, Indiana, USA \\ ${ }^{2}$ Birck Nanotechnology Center, West Lafayette, Indiana, USA \\ ${ }^{3}$ Michigan State University, East Lansing, Michigan, USA
}

\begin{abstract}
We have developed a scalable and rapid process for creating a low-cost humidity sensing transponder for wireless monitoring of moisture level within packaged goods. The sensor is composed of a moisture sensitive interdigitated capacitor connected to a planar spiral coil, forming a parallel LC circuit whose resonant frequency is a function of environment humidity. The fabrication process is composed of a series of laser processing steps performed on a commercially available metallized paper which included selective metal etching and welding using a Nd-YAG $(1.06 \mu \mathrm{m})$ laser and cutting the paper substrate by a $\mathrm{CO}_{2}(10.6 \mu \mathrm{m})$ laser. Selective laser ablation of the $10 \mu \mathrm{m}$ laminated $\mathrm{Al}$ film on the paper substrate was found to provide a unique one-step patterning of the conductive film on the paper substrate into the desired electrical patterns while simultaneously creating high surface area $\mathrm{Al}_{2} \mathrm{O}_{3}$ nanoparticles within the ablated regions. The intrinsic humidity-responsive characteristics of the laser induced metal oxide nanostructures provided a unique humidity sensitivity to the wireless sensor with a 10 folds higher performance than a similar LC resonant sensor that was prepared by conventional photolithography-based processes on a PCB substrate. The frequency change of the sensor was observed to be a linear function of $\mathrm{RH}$ (within the range of $0-85 \% \mathrm{RH}$ ), with an average sensitivity of $-87 \mathrm{kH} / \mathrm{RH}$ and good reversibility and repeatability.
\end{abstract}

\section{INTRODUCTION}

Nearly one third of all food produced in the world for human consumption ( $\sim 1.3$ billion tons), worth US\$990 billion, is thrown away each year, due to improper packaging, random food deterioration, and spoilage[1], [2]. Smart packaging technologies with integrated sensors that can monitor the quality of the product and its package play a vital role in reducing food waste by maximizing the shelf life of products and informing consumers with accurate information about the packaged product[3][4]. One of the major causes of food spoilage is the presence of excess moisture in the package of both fresh (i.e. fruits and vegetables) and dried products. Moisture absorbent pads, sheets, and blankets are often used to soak up excess liquid from the fresh foods such as fish, meat, fruits, and vegetables. Dried products (i.e. cereal, oats and wheats) are often packed with protective atmosphere gases such as carbon dioxide $\left(\mathrm{CO}_{2}\right)$ and nitrogen $\left(\mathrm{N}_{2}\right)$ with the aim of extending the shelf life by minimizing chemical deterioration and possible growth of aerobic spoilage microbes inside the packaged food[5], [6]. While simple standardized expiration dates have been made based on the materials used in the packaging of food products, most of these "expiration dates" are based on cautious estimates and in most cases the food product can be consumed well after these dates[7]. Furthermore, the possibility of food spoilage due to damages in the package, usually is not taken into account [8]. Therefore, an ideal way to ensure the quality and safety of the product is to monitor the moisture content within the sealed package using wireless and remote sensing systems. Due to this importance, different remote humidity sensing concepts with potential usage in food packaging have been explored and reported over the past few years that have explored the use of different active and passive RF wireless humidity sensing [9]-[12]. However, the high cost of manufacturing and assembly has made them uneconomical for widespread commercial use in food industry. To address this need, we have developed a novel method of manufacturing low-cost, wireless, passive humidity sensor based on an inductive-capacitive resonant circuit made by all laser processing of a commercially available wrap pan lining paper (WPLP) used for food cooking purposes that is composed of laminated films of aluminum (Al) foil $(\sim 10 \mu \mathrm{m})$ and hydrophobic parchment paper $(\sim 60 \mu \mathrm{m})$. The presented laser processing technology allows the wireless sensors to be fabricated at an extremely low cost and is appropriate for disposable packaging application where production cost is crucial for the viability of the technology.

\section{SENSOR DESIGN AND WORKING PRINCIPLE}

Fig. 1 shows an illustration of the wireless humidity sensing device for in situ monitoring of packaged goods. The sensor, referred to as the LC sensor, consists of an interdigital capacitor (IDC) and a spiral inductor laser ablated onto the $10 \mu \mathrm{m} \mathrm{Al} \mathrm{film} \mathrm{on}$ a commercially available WPLP. The final assembled sensor is modeled as an LC tank resonant circuit. The resonant frequency for this circuit is expressed as

$$
f_{s}=(1 / 2 \pi) \times\left(L_{s} C_{s}\right)^{-0.5}
$$

where $L_{s}$ is the inductance of the spiral coil, $C_{s}$ is the total equivalent capacitance of the IDC and built-in stray capacitances induced by the spiral coil. The key humidity sensing element within this circuit is $C s$ which is strongly dependent on the dimension of its electrodes and the relative permittivity (dielectric constant) of the substrate. The change in RH, alters the effective dielectric constant of the substrate which in turn shifts the sensor's resonant frequency. This change in resonant frequency can wirelessly be interrogated with an external reader.

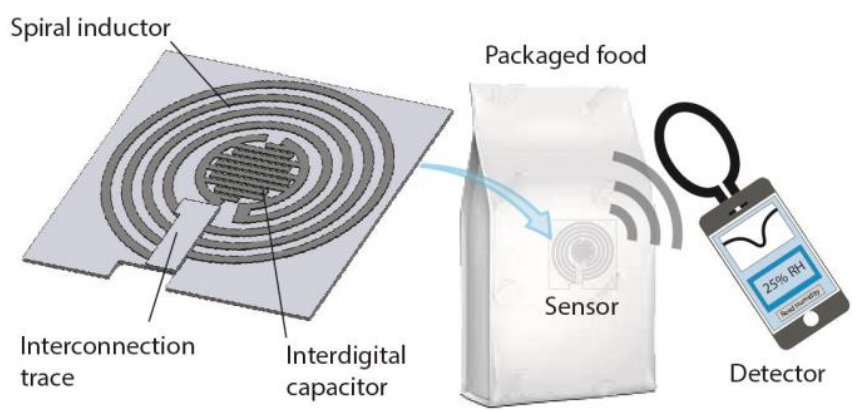

Figure 1: Design concept of a battery-less LC sensor that measures the humidity level inside a sealed package. The sensors resonant frequency can be remotely measured using an external coil connected to an RF reader. Hilton Head Island, South Carolina, June 3-7, 2018 


\section{DEVICE FABRICATION}

Fig. 2a-d illustrate the sequential processes used in the fabrication of the wireless humidity sensor. The fabrication process of the sensor was all performed by a computer-controlled laser engraver system (PLS6MW, Universal Lasers, Inc., Scottsdale, AZ) equipped with two lasers, a $\mathrm{CO}_{2}$ laser $(10.6 \mu \mathrm{m})$ and a $\mathrm{Nd}$ :YAG fiber laser $(1.06 \mu \mathrm{m})$. Before the start of fabrication process, the aluminum coating of the paper was wiped with an IPA solution and rinsed with DI water, followed by a nitrogen drying step. The WPLP was attached to a $1 \mathrm{~mm}$ glass substrate using scotch tape (3M) to provide easy handing while also ensuring that the paper substrate was always within the focal point of the laser throughout the fabrication steps. Next, the IDC and spiral coil were laser ablated onto the surface of the aluminum film using the $\mathrm{Nd}$ :YAG pulsed laser with pulse duration and repetition frequency set at $10 \mathrm{~ns}$ and $30 \mathrm{kHz}$, respectively. The operating power of $18 \mathrm{~W}$ and scanning speed $1.6 \mathrm{~m} / \mathrm{s}$ were optimized to completely remove the aluminum layer while causing minimum damage to the paper substrate. After completing the selective laser removal, the $\mathrm{CO}_{2}$ laser with power and speed settings of $10.5 \mathrm{~W}$ and $1.6 \mathrm{~m} / \mathrm{s}$ was used to cut through the paper substrate to release the sensor and its interconnection trace from the bulk WPLP film. At this point, the indented part of the paper with the dashed laser cut was folded onto the interconnection trace of the sensor. The folded paper prevented the electrical shorting of the coil by providing an insulating barrier between the interconnection trace and underlying traces of the spiral coil. This simple folding mechanism eliminated the need for printing any passivation layers on the sensor. The interconnection part was then laid over the sensor with aligning the connection pads. Next, a temporary $1 \mathrm{~mm}$ glass slide was placed onto the device to secure the interconnection trace. The electrical connection between the IDC and inductor was completed by laser welding the two connection pads using the Nd:YAG laser set at the power and speed of $18 \mathrm{~W}$ and $1.6 \mathrm{~m} / \mathrm{s}$. The low optical absorption of the glass and paper substrate at $1.06 \mu \mathrm{m}$ allows the laser beam to pass through the top glass and paper and only be absorbed by the thin aluminum foils at the center. The laser beam provides a concentrated heat at the interface of the two conductive aluminum films and fuses them together. After the laser welding process, the top glass layer is removed and fabrication process is complete. Fig. $2 \mathrm{e}$ and $\mathrm{f}$ show the commercial WPLP film used in this process and also some examples of coils with different number of turns, as well as the final assembled wireless humidity sensor.
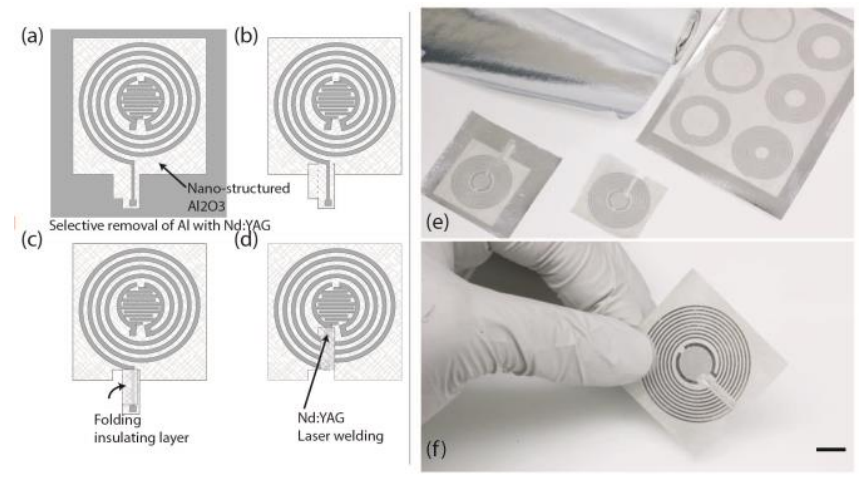

Figure 2: (a-d) Selective removal of Al and generation of nanostructured $\mathrm{Al}_{2} \mathrm{O}_{3}$ using $\mathrm{Nd}: Y A G$ laser, cut through the paper substrate using $\mathrm{CO}_{2}$ laser, folding insulating layer, laser welding to complete the resonant circuit (e,f) photograph of pristine and final wireless humidity sensor. Scale bar: $1 \mathrm{~cm}$

\section{RESULT AND DISCUSSION}

Optical and SEM images were used to evaluate the surface morphology and the effective removal of the Al layer from the WPLP substrate. Cross-section SEM images of before and after laser ablation show a clear removal of the conductive $10 \mu \mathrm{m} \mathrm{Al}$ layer with minimal damage to the $60 \mu \mathrm{m}$ underlying parchment paper substrate, Fig3 a, b. Fig. 3 c shows the SEM image of the laser ablated IDC sensor, composed of an array of $190 \mu \mathrm{m}$ wide Al electrodes with $25 \mu \mathrm{m}$ spacing. Close up SEM images of the laser ablated regions between the $\mathrm{Al}$ electrodes, reveal a clear change in the surface morphology with the generation of nanostructured $\mathrm{Al}_{2} \mathrm{O}_{3}$ particles on the fibrous surface of the paper, Fig. 3d. Static water contact angles on the pristine WPLP and Nd:YAG laser abated surface shows a significant decrease from $89.17^{\circ}$ to less than $11^{\circ}$ indicating the hydrophilic nature of the laser ablated region of the paper, Fig. 4a, b. High-resolution XPS spectra of the pristine Al surface shows the typical asymmetric Al2p peaks only observed for $\mathrm{Al}$ metal with the thin naive oxide layer. However, the Nd:YAG laser ablated samples showed a distinct signature of pure $\mathrm{Al}_{2} \mathrm{O}_{3}$ with a single symmetric peak within the $\mathrm{Al} 2 \mathrm{p}$ region with binding energy of $74.2 \mathrm{eV}$ and a full width at half maximum

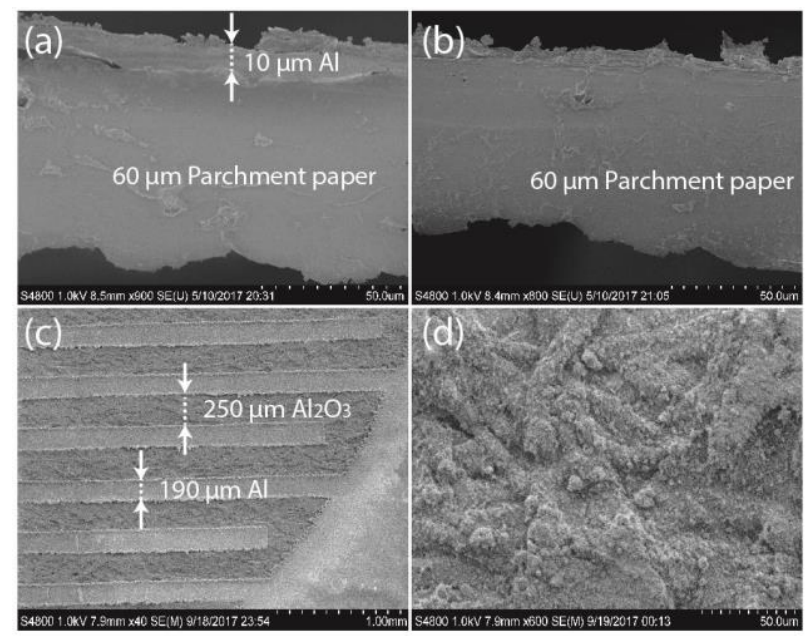

Figure 3: Cross-section SEM image of (a) before and (b) after selective laser removal of Al layer from WPLP (c) SEM image of laser ablated IDC, (d) high magnification of laser induced nano-structured $\mathrm{Al}_{2} \mathrm{O}_{3}$ on the surface of the paper substrate. (a)
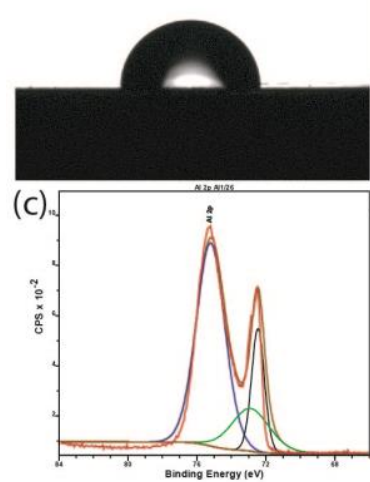

(b)
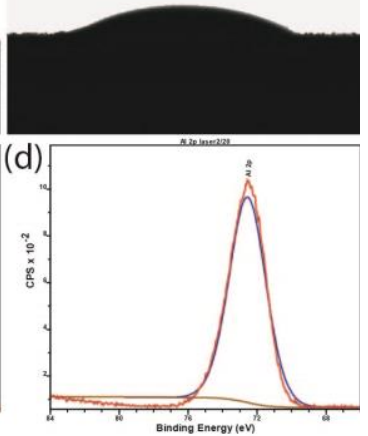

Figure 4: Static water contact angles on (a) pristine Al surface and (b) laser ablated Al surface, high resolution XPS spectra of Al2p of the Al layer (c) before and (d) after laser ablation with Nd:YAG laser 
of $1.3 \mathrm{eV}[13]$.

Fig. 5a shows a schematic of the setup used for characterizing the wireless humidity sensing performance of the LC sensor. An HP 4195A network/spectrum analyzer (HP/Agilent Technologies Inc., Santa Clara, CA) under standard settings was utilized in connection with an $8 \mathrm{~cm}$-diameter single turn coil made of standard 18 AWG insulated copper wire to serve as the external reader for wireless measurement of resonant frequencies using the phase dip technique. A computer was connected to the impedance analyzer via a GPIB interface and continuous data acquisition was implemented using custom designed Matlab-based program for visualization and data analysis. The sensor response to $\mathrm{RH}$ was characterized in a humidity chamber at a constant temperature $\left(21^{\circ} \mathrm{C}\right)$ while the relative humidity was changed from $0 \%$ to $85 \%$. The humidity level in the chamber was accurately measured with a commercial humidity sensor (Fluke 971). Humidity level within the chamber was controlled by the mixing ratio of dry and humidified $\mathrm{N}_{2}$ streams. The electrical parameters of the sensors including the inductance of the spiral coil and capacitance of the IDC components were first obtained through direct on-chip probing at $40 \% \mathrm{RH}$. The measured capacitance of the IDC sensor was $5.6 \mathrm{pF}$ and inductance of the spiral coil was $1.98 \mu \mathrm{H}$ which results is a calculated resonant frequency of $47.9 \mathrm{MHz}$. Fig. $5 \mathrm{c}$ (a) Inductive Coupling

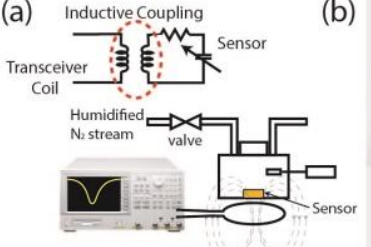

(c)

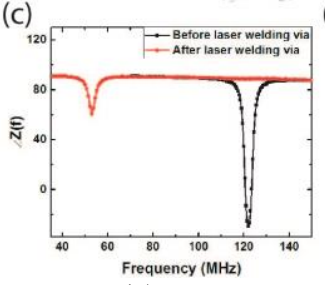

(b)

(d)

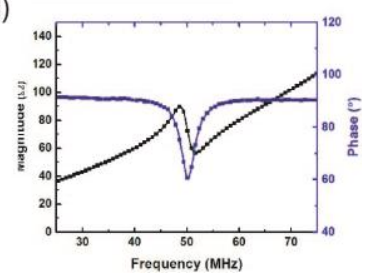

Figure 5: (a) Experimental setup, (b) optical image of $L C$ sensor and (c) impedance phase versus frequency before and after laser welding interconnection, (d) impedance phase and magnitude readings at the terminals of the readout coil coupled with the LC sensor at $40 \% \mathrm{RH}$ (a)
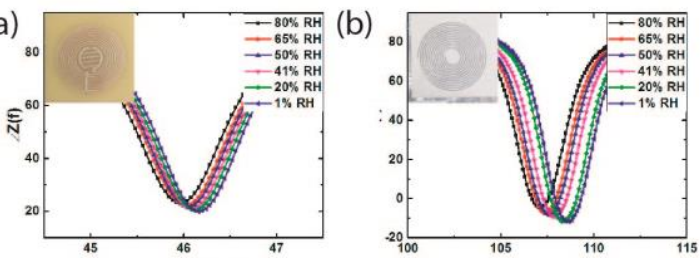

(c)
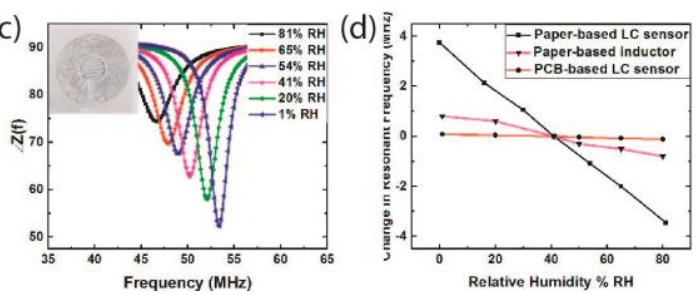

Figure 6: (a-d) Impedance phase vs freq. at different $R H$ for PCB LC resonant sensor, laser etched single inductor and LC resonant. shows the measured phase plot taken at a distance of approximately $3 \mathrm{~mm}$ between the sensor and readout coil before and after completing the circuit at $40 \% \mathrm{RH}$. As illustrated, the impedance phase dip decreased from $124 \mathrm{MHz}$ to $50.1 \mathrm{MHz}$ after connecting the IDC to the inductor. The $4 \%$ difference between calculated and experimental results through phase dip measurements can be explained by the errors that was introduced by parasitic capacitance of the electrical connections used in the on-chip probing measurements.

Fig. 6 shows the frequency sweeps of impedance phase of three different wireless humidity sensors in response to different humidity levels. The three sensors included a PCB-etched LC resonant sensor using standard photolithography-and wet etching processes, and a paper-based LC resonant sensor, and an inductor with 12-turns that were prepared by laser processing. The results show that the increases in RH will shift the phase dip towards lower frequencies in all three sensors. This indicated that the absorption of water vapor to all of the sensors substrates resulted in a decrease in resonant frequency. However, among all three sensors, the paper-based LC resonant sensors showed a greater shift in the location and size of the minimum phase with changes in $\mathrm{RH}$. The decrease in the dip size indicated a decrease in quality factor Q of the sensor due to the capacitive loss within the IDC at higher RH levels.

Fig. 6d plots the changes in resonant frequency of all three sensors as a function of RH. As the plot shows, the PCB etched LC resonant sensor had the lowest sensitivity $(-2.6 \mathrm{kH} / \mathrm{RH})$ which can be explained by low water vapor absorption sites on the active sensing area on the plastic substrate. However, the laser etched single inductor and LC resonant sensor on the WPLP showed a higher sensitivity to change in RH with the average sensitivity of 19.1 and $-87 \mathrm{kH} / \mathrm{RH}$, respectively. As expected, the high surface area of the laser induced $\mathrm{Al}_{2} \mathrm{O}_{3}$ nanoparticles provide considerably higher water vaper adsorption sites which evidently result in greater humidity sensing performance.

Fig. 7 shows the reversible changes in the resonant frequency of the paper-based LC sensor as the relative humidity was varied cyclically between $40 \%-0 \%$ and $40 \%-85 \%$ RH. The sensor showed a high degree of reversible performance at both high and low humidity levels. The response and recovery times under testing cycle from $40 \%$ to $0 \% \mathrm{RH}$ were $51 \mathrm{~min}$ and $44 \mathrm{~min}$, respectively. Although, during higher RH cycles from $40 \%$ to $85 \%$, the average response and recovery time were shorter, $9.6 \mathrm{~min}$ and $14.3 \mathrm{~min}$, respectively. The longer recovery phase from higher to lower $\mathrm{RH}$ can be explained by the fact that it takes a longer time for the water vapor to evaporate than to absorb on the $\mathrm{Al}_{2} \mathrm{O}_{3}$ nanostructure surface.

This phenomenon is prominent and slower in cases were moister is adsorbed in the form of water molecules within the inner walls and inside the $\mathrm{Al}_{2} \mathrm{O}_{3}$ nanostructure (cycles between 40 to $0 \% \mathrm{RH}$ ). However, at higher humidity level swings (40 to $85 \% \mathrm{RH}$ ), the
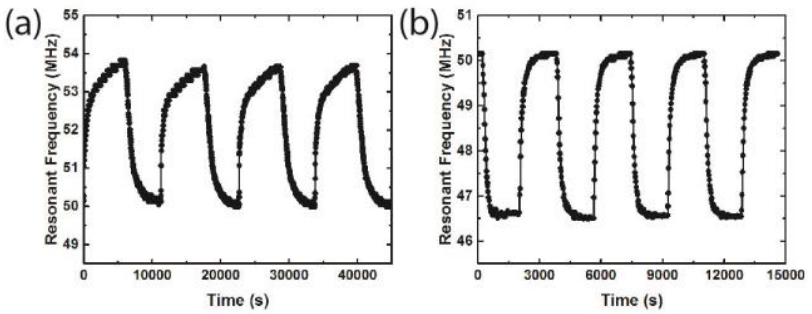

Figure 7: Cyclically varied $\mathrm{RH}$ between (e) $0 \%-40 \%$ and $(f)$ $40 \%-85 \%$. 

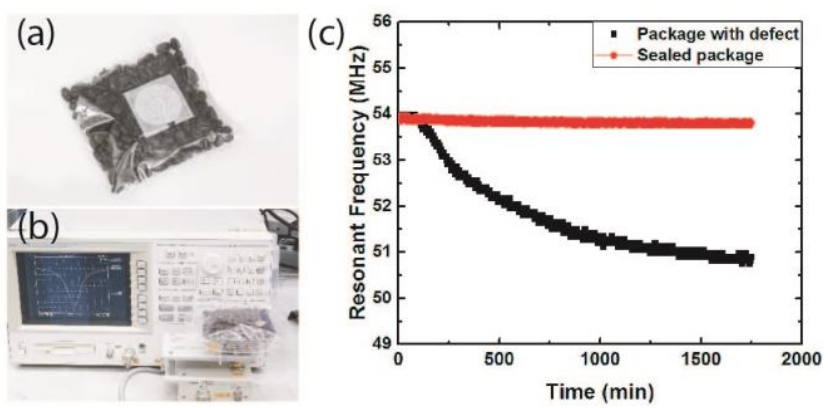

Figure 8: (a) LC sensor placed in coffee bean package (b) readout setup, (c) resonant frequency reading from well-sealed and defected package.

change in dielectric properties of the sensor is influenced by physical absorption of water molecules known as capillary condensation that take places within nanostructure pores of the sensor. Capillary condensation has a much faster attachment and removal from the surface. Therefore, the overall response and recovery time of the sensor is shorter at higher moister levels. Finally, as a proof of concept, the sensor was placed inside packaged roasted coffee beans that was filled with nitrogen gas, Fig. 8. As shown in Fig. 8c, the resonant frequency of the sensor in the well-sealed packaging remained stable at $53.8 \mathrm{MHz}$. After perforating the package with a sharp needle, the resonant frequency slowly decreased to $50.1 \mathrm{MHz}$ due to water vapor adsorption into the package.

\section{CONCLUSIONS}

We have developed an inexpensive and scalable fabrication technique for manufacturing a wireless paper-based humidity sensor for monitoring food packaging quality. The sensor was made of a planar inductor-capacitor resonant circuit with a resonant frequency that is dependent on the environment moisture. The sensor's resonant frequency was wirelessly interrogated through an inductive coupling link with an external reader. The sensor was made by an all laser processing of a commercial WPLP with a $10 \mu \mathrm{m}$ laminated $\mathrm{Al}$ foil. In this study, we established the ability to selectively remove the conductive Al layer while concurrently generate high-surface area $\mathrm{Al}_{2} \mathrm{O}_{3}$ nanostructures within the ablated regions using $\mathrm{Nd}$ :YAG laser radiation under ambient conditions. The intrinsic humidity-responsive properties of laser induced $\mathrm{Al}_{2} \mathrm{O}_{3}$ nanostructures provided a unique and relatively high sensitivity to the laser etched LC sensors as compared to wet etched PCB-based LC sensor with similar design and dimensions. This work might inspire both industrial and scientific communities to reexamine the potential use of the presented laser processing approach in scalable production of future low-cost energy storage and bio-sensing devices.

\section{REFERENCES}

[1] M. F. Bellemare, M. Çakir, H. H. Peterson, L. Novak, and J. Rudi, "On the Measurement of Food Waste," Am. J. Agric. Econ., vol. 99, no. 5, pp. 1148-1158, Oct. 2017.

[2] K. L. Thyberg and D. J. Tonjes, "Drivers of food waste and their implications for sustainable policy development," Resour. Conserv. Recycl., vol. 106, pp. 110-123, Jan. 2016.

[3] K. B. Biji, C. N. Ravishankar, C. O. Mohan, and T. K. Srinivasa Gopal, "Smart packaging systems for food applications: a review," J. Food Sci. Technol., vol. 52, no. 10, pp. 6125-6135, Oct. 2015.

[4] J. P. Kerry, M. N. O'Grady, and S. A. Hogan, "Past, current and potential utilisation of active and intelligent packaging systems for meat and muscle-based products: A review," Meat Sci., vol. 74, no. 1, pp. 113-130, Sep. 2006.

[5] A. R. Davies, "Advances in modified-atmosphere packaging," in New Methods of Food Preservation, Boston, MA: Springer US, 1995, pp. 304-320.

[6] I. S. Arvanitoyannis and A. C. Stratakos, "Application of Modified Atmosphere Packaging and Active/Smart Technologies to Red Meat and Poultry: A Review," Food Bioprocess Technol., vol. 5, no. 5, pp. 1423-1446, Jul. 2012.

[7] A. Giménez, F. Ares, and G. Ares, "Sensory shelf-life estimation: A review of current methodological approaches," Food Res. Int., vol. 49, no. 1, pp. 311-325, Nov. 2012.

[8] M. G. Corradini, "Shelf Life of Food Products: From Open Labeling to Real-Time Measurements," Annu. Rev. Food Sci. Technol., vol. 9, no. 1, pp. 251-269, Mar. 2018.

[9] R. A. Potyrailo, N. Nagraj, Z. Tang, F. J. Mondello, C. Surman, and W. Morris, "Battery-free Radio Frequency Identification (RFID) Sensors for Food Quality and Safety," J. Agric. Food Chem., vol. 60, no. 35, pp. 8535-8543, Sep. 2012.

[10] T. J. Harpster, S. Hauvespre, M. R. Dokmeci, and K. Najafi, "A passive humidity monitoring system for in situ remote wireless testing of micropackages," $J$. Microelectromechanical Syst., vol. 11, no. 1, pp. 61-67, 2002.

[11] a. D. DeHennis and K. D. Wise, "A wireless microsystem for the remote sensing of pressure, temperature, and relative humidity," J. Microelectromechanical Syst., vol. 14, no. 1, pp. 12-22, 2005.

[12] A. V. Quintero, F. Molina-Lopez, E. C. P. Smits, E. Danesh, J. van den Brand, K. Persaud, A. Oprea, N. Barsan, U. Weimar, N. F. de Rooij, and D. Briand, "Smart RFID label with a printed multisensor platform for environmental monitoring," Flex. Print. Electron., vol. 1, no. 2, p. 025003 , 2016.

[13] T. Song, Q. Liu, J. Liu, W. Yang, R. Chen, X. Jing, K. Takahashi, and J. Wang, "Fabrication of super slippery sheet-layered and porous anodic aluminium oxide surfaces and its anticorrosion property," Appl. Surf. Sci., vol. 355, pp. 495-501, Nov. 2015.

\section{CONTACT}

*B. Ziaie, bziaie@purdue.edu 